\title{
Key Science Observations of AGNs with KaVA Array
}

\author{
Motoki Kino* \\ Korea Astronomy and Space Science Institute (KASI), 776, Daedeokdae-ro, Yuseong-gu, \\ Daejeon, Republic of Korea 305-348 \\ E-mail: kinodkasi.re.kn
}

\section{Kotaro Niinuma}

Graduate School of Science and Engineering, Yamaguchi University, Yoshida 1677-1,

Yamaguchi, Yamaguchi 753-8512, Japan

\section{Guang-Yao Zhao, Bong Won Sohn}

Korea Astronomy and Space Science Institute (KASI), 776, Daedeokdae-ro, Yuseong-gu, Daejeon, Republic of Korea 305-348

\section{KaVA AGN Sub Working Group}

\begin{abstract}
KaVA (KVN and VERA Array) is a new combined VLBI array with KVN (Korean VLBI Network) and VERA (VLBI Exploration of Radio Astrometry). First, we briefly review the imaging capabilities of KaVA array which actually achieves more than three times better dynamic range than that achieved by VERA alone. The KaVA images clearly show detailed structures of extended radio jets in AGNs. Next, we represent the key science program to be led by KaVA AGN sub working group. We will conduct the monitoring observations of Sgr A* and M87 because of the largeness of their central super-massive black hole angular sizes. The main science goals of the program are (i) testing magnetically-driven-jet paradigm by mapping velocity fields of the M87 jet, and (ii) obtaining tight constraints on physical properties of radio emitting region in Sgr A*.
\end{abstract}

12th European VLBI Network Symposium and Users Meeting,

7-10 October 2014

Cagliari, Italy

\footnotetext{
* Speaker.
} 


\section{Introduction}

Active galactic nuclei (AGN) occasionally produce powerful and collimated jets of magnetized relativistic particles which can extend beyond galactic scales, and have an impact on galaxy evolutions. Although magnetic driven scenario of relativistic jets is widely discussed (e.g., [四; [■]]), there is no conclusive observational evidence to prove it. In order to explore jet formation processes by a central engine composed of a supermassive black hole and accreting matter onto it, Very Long Baseline Interferometer (VLBI) is one of the most powerful tools because they can probe innermost regions of relativistic jets with its high spatial resolution.

Recently, a new VLBI facility, named KaVA, consisting of Korean VLBI network (KVN) and VLBI Exploration of Radio Astrometry (VERA) has been constructed in East Asia region (http://veraserver.mtk.nao.ac.jp/). KVN is the first VLBI array dedicated to the mm-wavelength radio observations in East Asia operated by Korean Astronomy and Space Science Institute (KASI) ([3] ) (http://kava.kasi.re.kr/kava main.php). KVN consists of three 21-m-diameter radio telescopes: one in Seoul, one in Ulsan, and one on Jeju Island, Korea. In this proceedings, AGN Key Science Project (KSP) with KaVA, i.e., monitoring M87 and Sgr A* at $23 \mathrm{GHz}$ and $43 \mathrm{GHz}$ is summarized.

\section{Imaging Capability of KaVA}

Here we briefly review imaging capability of KaVA. In radio interferometers, the detection limit (equivalent to thermal noise level) of images is given by $\sigma_{\mathrm{th}}=\frac{2 k_{\mathrm{B}} T_{\mathrm{sys}}}{A_{\mathrm{eff}} \eta_{\mathrm{q}} \sqrt{N_{\text {ant }}\left(N_{\mathrm{ant}}-1\right) B W t_{\text {int }}}}$ where $A_{\text {eff }}, \eta_{\mathrm{q}}, T_{\text {sys }}, N_{\text {ant }}, B W, t_{\text {int }}$, are the efficient aperture area of the antennas, the quantization loss factor, the system temperature, the number of antennas, bandwidth, and the total integration time, respectively. When using natural weighting, a typical thermal noise for KaVA observation of M87 at $23 \mathrm{GHz}$ with $B W=32 \mathrm{MHz}$ is $\sigma_{\mathrm{th}} \approx 0.7 \mathrm{mJy}$ beam $^{-1}$ and the corresponding signal-to-noise ratio (SNR) is $\sim 1400$ (see details for [四]).

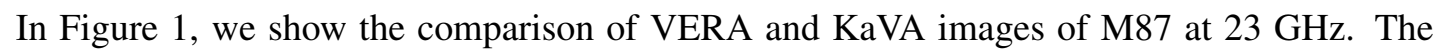
higher dynamic range of KaVA enables us to obtain the extended structure of the M87 jet up to 10 mas scale. Qualities of VLBI images tend to be limited by their dynamic ranges rather than SNR. The dynamic range of VLBI images can be given by $\frac{I_{p}}{\sigma_{\text {im }}}=\frac{\sqrt{M_{\text {scan }}} \sqrt{N_{\text {ant }}\left(N_{\text {ant }}-1\right)}}{\max [\varepsilon, \Delta \phi]}$ where $M_{\text {scan }}, \varepsilon$, and $\Delta \phi$ are the number of scans for each observation, and degrees of amplitude and phase errors, respectively. The dynamic range of KaVA image in Figure $\mathbb{D}$ reaches $\sim 1000$ and it is more than three times better than that achieved by VERA alone ([䧃]).

\section{Key Science Project}

\subsection{M87}

M87, a nearby giant radio galaxy located at a distance of $16.7 \mathrm{Mpc}$, hosts one of the most massive super-massinve black holes with it mass of $6 \times 10^{9} M_{\text {sun }}$. Thanks to its proximity and the largeness of its central black hole, M87 is well known for being the best source for imaging the innermost part of the jet base (e.g., [G] ). According to the leading scenario of jet formation model, a jet is thought to be powered by a central engine in a highly magnetized state, and accelerated via 

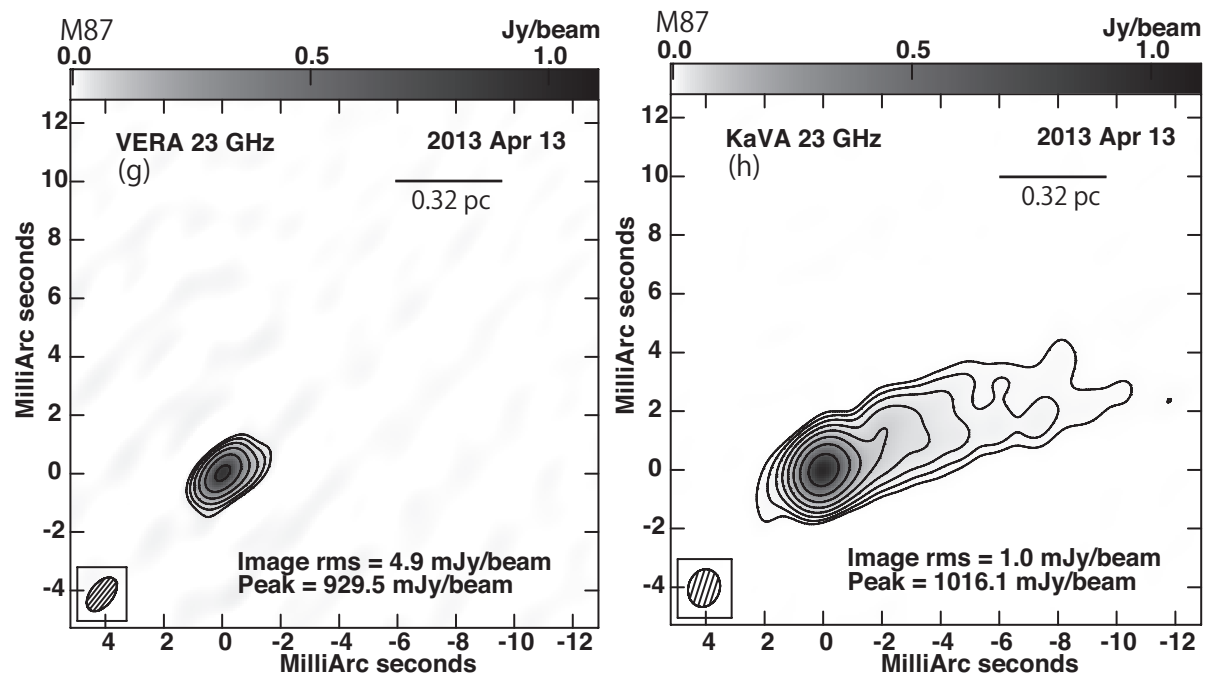

Figure 1: The comparison of VERA and KaVA images of M87 at $23 \mathrm{GHz}$.

conversion of magnetic energy into kinetic one. Relativistic magnetohydrodynamic models have suggested that jets are gradually accelerated on a scale which can be well observed by VLBI in the case of M87 (e.g., [[]]). Hence, it is possible to constrain such models by comparing the modelpredicted velocity fields and observed one. Indeed, mapping the apparent velocities of the M87 jet has been explored in previous work [6]. However, the reported apparent velocities at 1-10 mas from the central engine are significantly different and it is controversial. The aim of this KSP is measuring the actual velocity field in the M87 jet to test magnetically-driven jet paradigm with sufficiently short interval to avoid possible component mis-identifications.

\subsection{Sagittarius $A^{*}$}

The center of Milky Way hosts Sgr A*, a massive black hole with the largest angular size, hence it is one of the best laboratories to explore ultimate vicinity of black holes (e.g., [四]). During the past VLBI monitoring of Sgr A* at $43 \mathrm{GHz}$, the flare phenomena was found in 2007 [8]. Interestingly, the size of the major axis remained the same while the flux increased about $2 \mathrm{Jy}$. This is the first report for VLBI scale flare and we do not know how frequently it happens. In Figure ■, we present the $u, v$ coverage and the preliminary image of $\mathrm{Sgr} \mathrm{A}^{*}$ at $43 \mathrm{GHz}$ with the bandwidth of $256 \mathrm{MHz}$. We emphasize that KaVA can achieve a good performance for Sgr A* observations, since it contains more short baselines than other VLBI arrays. Short baselines in KaVA provide more effective sampling of the visibilities of Sgr $\mathrm{A}^{*}$ than VLBA and it enables us to get better measurements of the source size. Accurate measurements of size and flux of Sgr A* by KaVA at $43 \mathrm{GHz}$ will enable us to give tight constraints on physical quantities in Sgr A* such as magnetic field strength since these quantities have strong dependences on the source size [Q]. 

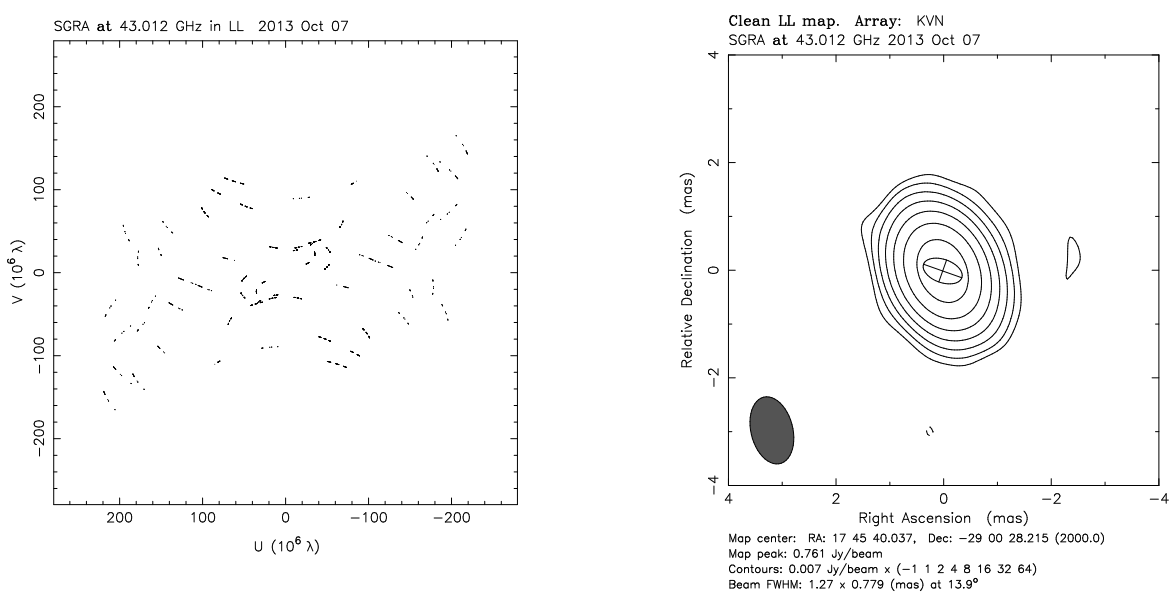

Figure 2: Left: The $u, v$ coverage of KaVA observation of Sgr A* at $7 \mathrm{~mm}$ performed on Oct 7th 2013. Right: Corresponding KaVA image of Sgr A* with the best-fit elliptical Gaussian model.

\section{References}

[1] R. D. Blandford, \& R. L. Znajek, Electromagnetic extraction of energy from Kerr black holes, MNRAS, 179, 433 (1977)

[2] J. C. McKinney, General relativistic magnetohydrodynamic simulations of the jet formation and large-scale propagation from black hole accretion systems, MNRAS, 368, 1561 (2006)

[3] S.-S. Lee, L. Petrov, D.-Y. Byun et al. Early Science with the Korean VLBI Network: Evaluation of System Performance, AJ, 147, 77 (2014)

[4] K. Niinuma, S.-S. Lee, M. Kino et al. VLBI observations of bright AGN jets with KVN and VERA Array (KaVA): Evaluation of Imaging Capability, PASJ, in press (2015)

[5] K. Hada, A. Doi, M. Kino et al. An origin of the radio jet in M87 at the location of the central black hole, Nature, 477, 185 (2011)

[6] K. Asada, M. Nakamura, A. Doi, H. Nagai, \& M. Inoue Discovery of Sub- to Superluminal Motions in the M87 Jet: An Implication of Acceleration from Sub-relativistic to Relativistic Speeds ApJL, 781, LL2 (2014)

[7] S. S. Doeleman, J. Weintroub, A. E. E. Rogers et al. Event-horizon-scale structure in the supermassive black hole candidate at the Galactic Centre, Nature, 455, 78 (2008)

[8] K. Akiyama, R. Takahashi, M. Honma, T. Oyama, \& H. Kobayashi, Multi-Epoch VERA Observations of Sagittarius A*. I. Images and Structural Variability, PASJ, 65, 91 (2013)

[9] M. Kino, F. Takahara, K. Hada, A. Doi et al. Relativistic Electrons and Magnetic Fields of the M87 Jet on the 10 Schwarzschild Radii Scale, ApJ, 786, 5 (2014) 